\title{
Meeple Centred Design: A Heuristic Toolkit for Evaluating the Accessibility of Tabletop Games
}

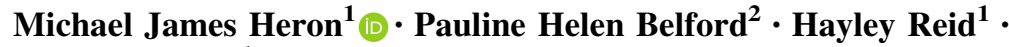 \\ Michael Crabb ${ }^{1}$
}

Received: 18 January 2018/Accepted: 15 April 2018/Published online: 27 April 2018

(C) The Author(s) 2018

\begin{abstract}
Evaluation of accessibility within a tabletop context is much more complicated than it is within a video game environment. There is a considerable amount of variation in game systems, game mechanisms, and interaction regimes. Games may be entirely verbal, or completely non-verbal. They might be real-time or turn based, or based on simultaneous actions. They can be competitive or cooperative, or shift from one to the other during a single game session. They might involve visual pattern recognition or force players to memorise game state without visual cues. They may involve touch, or smell. They might involve social deduction or betrayal. They can encompass all sensory faculties, in differing degrees. Almost all games have accessibility considerations that should be taken into account, but there is currently no comprehensive tool by which this can be done that encompasses the rich variety of tabletop gaming interaction metaphors. In this paper, the authors discuss the heuristic lens that is used by the Meeple Centred Design tabletop accessibility project. This is a tool that has been applied to one hundred and sixteen games to date, and the full results of these have been published for analysis and consideration within the wider tabletop gaming community.
\end{abstract}

Michael James Heron

m.j.heron1@rgu.ac.uk

Pauline Helen Belford

p.belford@dundeeandangus.ac.uk

Hayley Reid

1300777@rgu.ac.uk

Michael Crabb

m.j.crabb@rgu.ac.uk

1 Robert Gordon University, Aberdeen AB10 7QB, Scotland, UK

2 Dundee and Angus College, Arbroath DD11 3EA, Scotland, UK 
Keywords Board games · Accessibility · Inclusion · Tabletop · Meeple

Like Us · Universal access

\section{Introduction}

The playing of games is an important source of social capital (Heron 2012, 2016; Lenhart et al. 2008) and a pre-requisite for the effective integration of individuals into the cultural economy. Video games in particular are a vibrant element of the modern recreational landscape. The highest profile titles have an equivalent sociocultural impact as blockbuster movies, chart-topping albums, and successful books. Within the field of game studies, the accessibility of gaming titles has often been addressed (c.f. Heron 2012, 2015; Di Loreto et al. 2013; Westin and Dupire 2016) although perhaps in practise it is more honoured in the breach than in the observance (Heron 2012). Recent releases, such as Naughty Dog's 'Uncharted 4' have shown though that accessibility can be a mainstream issue, and that adherence to accessible design can generate wide-spread praise and recognition. This bodes reasonably well for the industry.

However, parallel to the growth of video games as important cultural artefacts is a modern Renaissance in traditional tabletop games. These games do not have the mainstream impact of their digital cousins, and suffer from significant historical baggage when they are introduced to new players. Nonetheless, there has been a substantial rise in the cultural penetration associated with the new wave of what are sometimes known as 'designer board games'. To go with this, there has been a marked increase in the number of players, critics and advocates within this relatively obscure area of game culture. This has in recent years led to a degree of recognition of the hobby in the wider area of game studies. The digitally focused Game Developer Conference (GDC) has offered co-located space for the discussion and presentation of tabletop games. Firaxicon, a convention for fans and developers of Firaxis video games, included space for its licenced and related tabletop game off-shoots. This is in addition to the remarkable growth in attendance at dedicated tabletop gaming conventions such as Origins, Gencon, BGGCon, and the UK Games Expo.

As the popularity of the hobby increases, so too does the scope for accidental exclusion of marginalised groups. Many games on the market are inaccessible to those with visual, physical, cognitive or communicative impairments. Often inaccessibility is an unavoidable consequence of game design decisions. Games which enforce dexterity or tactility of components as core game elements are unlikely to ever be accessible to those with physical impairments, as an example. However, there is a much more pervasive core of inaccessibility as a result of component choice, optional aesthetic considerations, and the type and number of individual tokens available in the box.

In this paper we discuss the Meeple Like Us project. This project is focused on mapping out the accessibility landscape of tabletop gaming. The project has a focus on examining not only the obvious issues but also those that stem from the intersection of compounding impairments. The core of this project is a heuristic 
evaluative toolkit that has been applied to date to one hundred and twenty relatively high-profile table-top games. From this, a comprehensive accessibility teardown has been written for each. We present the heuristic framework in this paper with reference to some specific examples of inaccessibility in tabletop games. We argue that there should be greater inclusion of diversity when play-testing game titles, but hope this framework serves as a useful preliminary exercise for game designers and developers.

\section{Sociocultural Importance of Board Games}

Full participation in popular culture offers an important opportunity for people to build cultural capital and socially integrate with others in a community (Lee et al. 2015; Willekens and Lievens 2014). For youth in particular, cultural literacy involves a wide-variety of transmedia products, encompassing movies; music; novels; video games; and board games. Appreciation of modern popular cultural products is no longer simply a passive act of consumption. Instead it is a process of individual and communal construction through interpretation, debate and an emergence of collaboratively constructed critical perspectives (Fiske 2010; Harrison and Barthel 2009). Appreciation of popular cultural products facilitates the building of common conversational ground, and permits friendships to accrete around a set of shared cultural experiences.

It is not however the case that all individuals have equal opportunity to participate in this process. Many cultural products, popular or otherwise, remain either physically or sociologically inaccessible to large segments of the population. For some, this may be a case of simply not seeing how cultural elements have value. For others, the value is apparent but the products themselves are exclusionary because they do not, or cannot, take into account physical, cognitive and socioeconomic accessibility.

Most industries have made considerable effort to increase the accessibility of their products, for example with close-captioned movies or television shows, audiobooks, or braille documents. Gaming products though remain in many cases obstinately inaccessible (Heron 2012). The difficulties and complexities of developing accessible video games are now receiving some attention from the industry. Progress is being made, albeit at too slow a rate to generate great enthusiasm. However, gaming as a recreational activity encompasses a wider range of forms than popular video games. There exists a vibrant, and growing, renaissance in the area of tabletop board and card games. The past 20 years have been the best years that the industry has known in terms of economic impact and popular appreciation.

The stuffy reputation of board games as being centred around old family classics such as Monopoly, Cluedo and Risk is being replaced by a marketplace of innovative and impeccably designed modern titles with a distinctly international feel and appeal. Games such as Settlers of Catan, Carcassonne, Pandemic, Ticket to Ride, and Dominion have each spawned dedicated followers of fans. They have inspired hundreds of talented designers to focus on developing new titles and 
innovating upon the ones that have come before. Buoyed by relatively easy access to start-up capital through crowdfunding platforms such as Kickstarter (Roeder 2015), the hobby game market in 2014 swelled to an estimated $\$ 880 \mathrm{~m}$ in sales in the US alone. Almost $\$ 200 \mathrm{~m}$ of that was directly linked to board games (ICV2 2015). It is difficult to truly put a figure on the current size of the industry, but there is every reason to expect that it will continue to grow by double digits on a yearly basis for the foreseeable future.

Board gaming represents one of the easiest ways for groups of people to sit down and enjoy a shared gaming experience with considerable opportunity for building social capital between friends and relatives. There is a growing body of evidence too that suggests beyond offering opportunities for friends and family to enjoy one another's company, gaming (and board gaming in particular) offers an effective avenue to explore play therapy interventions (Carroll 2002; Hromek and Roffey 2009; Pon 2010). Numerous popular web series act as advocates and curators of this culture, helping to explore both the social and ludic implications of game design choices and opportunities. In most cases, they are being collaboratively crowdfunded to do so in exchange for little more than the value their viewers perceive in the commentary they offer. In almost every metric one might choose to measure it, this industry is currently engaged in a long-lasting and feasibly sustainable golden age. Accessibility as a concept often focuses on the day to day realities of functional living. We argue that access to the products of culture and the means to satisfying recreation is every bit as important in enabling a genuinely inclusive society.

\section{Accessibility of Board Games}

Board gaming lags considerably behind even video gaming when it comes to the accessibility of the products that are on the market. Accessibility is a multi-channel challenge where subtle and nuanced interactions of impairments may create complications as a result of intersectionality (Heron et al. 2013). This means that there is rarely a single correct compensation, especially when considered within the context of the complexity of formal rules-based systems such as board games.

For those with physical impairments, it is often simply not possible for them to fully engage with a game without the intervention of a third party. Most board games are physical products, with spatially complex game-states and multiple, often small, components that are interrelated in often subtle ways. The location of components on a game board may be significant, and the margin of error for meaningful positioning may be very limited. Some components may be very small and fiddly, occasionally difficult for even unimpaired players to effectively handle without frustration. A standard card-game may require multiple players, each managing multiple decks of cards, with some communal representation of gamestate between them. Some games require the physical flipping of game tiles, and the layering of visual markers on these as the game state changes. Others require a constant stream of collection and discarding of small cubes, and the placement of miniature people (known in board gaming jargon as 'Meeple') within a spatially complex and state-dependent representation of a fantasy city. 
Some games require the ability to take a long term view from an elevated position to consider the strategic implication of decisions. Such games can be played, with difficulty, by those with impairments provided they have people around them willing to compensate for interaction difficulties. Such interventions can be slow; be difficult to perform; or simply interrupt the flow of gameplay. More importantly, they do not permit the impaired player to interact with the game themselves, restricting autonomy and the empowered wish fulfilment that is a core feature of many titles. Some games require a player to have cards or resources that are hidden from others-in such games, an impaired player either cannot fully participate or must be supported by another individual who cannot play the game. A game as common as poker cannot be fairly played if one's cards are known by another active participant.

Game-states are often represented in heavily thematic ways. This disadvantages those with visual impairments. Font choice may be sub-optimal for readability, or the sheer number of choices on a board may require text size to be uncomfortably small. Many games involve lots of components, some of which may be colour coded, or difficult to make out against the backdrop of the game-board. The physical orientation of pieces played on a board may have special meaning, so even if symbols can be visually ascertained, they may have subtleties of positioning that complicate interpretation. Cards may have considerable amounts of text, densely written and full of complicated instructions. Again, these do not prevent someone from playing if they have others around them willing to compensate, but in doing so it moves the responsibility from the game developers onto the players, and risks much of the flow that comes from effortless and absorbing game-playing.

Those with cognitive impairments encounter more problems-rules may be complicated or heavily state dependent, resulting in complex compound conditionals such as 'If the villain has this card in their deck, then all attacks of this particular type are at +1 for this round only, but if they have a different card then all damage they do is at -1 unless it's an electricity based attack'. Such rules are difficult enough for most of us to fully understand. In some games where the rules themselves are modifiable familiarity with the base rule-set will not meaningfully simplify the cognitive cost of participation. Similarly, game-state itself might be complicated-even if the rules are simple, the end stages of a game may involve highly interrelated physical state, where ascertaining the best way to contain an escalating situation is part of the logic puzzle involved. Many games embrace failure as a likely outcome, which can add an additional level of frustration for those that feel already disempowered by complexity. Most require specific symbols, locations and orientations to have particular meaning within the game-state, adding a burden of recall on the player to understand not just the game-state itself, but the abstract mechanism that exists for representing it.

Those with hearing impairments are perhaps least affected by board game design, but some games have accompanying soundtrack CDs which provide gamedependent cues to which all players must respond.

There exist a variety of accessible versions of games such as Chess and Monopoly. These are well-meaning but at best a partial solution to the problem because they do not address the issue of full participation in popular culture. 
Accessible versions of chess, Monopoly and Scrabble are welcome but limiting people to these titles severely restricts the scope of participation in board gaming culture for those with impairments. There exist some bespoke projects to offer accessible versions of modern board game titles. However, such solutions are also only partial because they are of use primarily to the blind, and only to the subset of blind players (estimated by the National Federation of the Blind to be around 10\% of the legally blind population) that are conversant with braille. Even within this limited solution space, the nature of braille means that certain long text passages would not be possible to convert into a tactile format within the narrow confines of a physical token or card. Advice within the gaming community for those with impairments tends to focus on 'Do it yourself' hacks and compensations. That can work as an individual solution to an individual problem but lack generalisability and ease of support.

Accessibility is about removing the barriers that stop people playing. Those barriers are sometimes physical, sometimes philosophical, sometimes economic, and sometimes in terms of inclusiveness of representation. A large portion of the teardowns performed by Meeple Like Us are about disabilities. Accessibility though is a much broader topic than that and it's important that a review of how accessible a game is takes this into account with the same degree of seriousness as the other barriers.

A real, lasting solution must involve raising everyone to the same standard rather than attempting to create different, incompatible categories of accessible games for those with impairments, or to focus on ineffective compensation strategies such as braille dice or overlays.

However, this is a complex challenge.

The 'feel' of a game is important, and thematics are an important aspect of that feel. A game based around pretending to be superheroes for example has an enhanced feel as a result of cards being ornamented with comic-book art and fonts. We cannot address accessibility issues by ignoring the importance that theme plays in the overall experience.

Board game developers are already in many ways committed to particular designs, thematics and rule-sets. We cannot address the issues by relying on developers to redesign their games so that they are fully accessible, at least until a sufficient business case can be made for accessibility as a valuable market opportunity.

Mapping out the contours of this topic is an important first step in being able to offer a meaningful strategy for raising the floor on accessible design in board games. What's needed is a programme of research aimed at examining potentially external sources of accessibility compensation such as digital apps, dedicated cognitive support tools, and learning aids and tutorials that come from outside the box of the game itself. Before that work can begin, we must first understand the nature of the problem. There are many ways that a topic like this can be addressed, including via the ecology of human performance (Dunn et al. 1994) and the person-environment occupation model (Law et al. 1996). These however are outside the scope of this specific paper which is focused primarily on the specific intersections of player and game design. Future work will likely draw more heavily from insights permitted via these techniques. 


\section{The Meeple Centred Design Heuristic Toolkit}

As part of the process of mapping out the accessibility landscape of tabletop games, the Meeple Like Us project has begun a long process of publishing comprehensive accessibility teardowns of popular titles. These may be found at the website http:// meeplelikeus.co.uk. Behind the scenes of the published posts on this topic is a heuristic toolkit. This has been developed to offer lenses through which qualified researchers might focus on the key elements of difficulty to be found in tabletop interaction. This toolkit is broken into six key sections:

1. Visual impairments, including colour blindness.

2. Cognitive impairments, focusing on fluid and crystalised intelligence.

3. Physical impairments, in both gross and fine-grained motor skills.

4. Communication impairments, relating to the ability to speak, hear or otherwise communicate.

5. Socioeconomic impairments, with regards to cultural inclusion and economic considerations.

6. Intersectional issues, in which particular combinations of impairment may have additional impact.

The toolkit is presented as a series of topic for contemplation-not all will be relevant to all games, and the context of the game itself may introduce complexities that the toolkit itself cannot be generalised to accommodate. It requires significant understanding of the subtle interrelationship of accessibility concerns (Heron et al. 2013) and a familiarity with the game in question that can only come from direct or observed experience. As such, it is not a tool that could yet be adopted for a general audience in ascertaining the accessibility issues of board games. It does however offer an opportunity to raise awareness of the kind of issues that may be encountered.

With this framework we focus only on symptoms and not on root causes. We make no assumption as to the condition which creates the circumstances under which impairments may manifest. Every individual is different in terms of how their personal conditions interact with their own personal context. Each Meeple Like Us teardown offers a discussion and a recommendation, but this recommendation must be assessed for individual applicability in light of the specific issues outlined. The full list of Meeple Like Us teardowns and the results may be found online. ${ }^{1}$

The toolkit is in part a recommendation regime, designed to allow the Meeple Like Us project to make meaningful suggestions as to what games people may want to avoid, and which they may be surprised to know they can effectively play. It is also in part a tool for offering meaningful suggestions on what must be considered when improving the general accessibility of tabletop games.

There are significant logistical concerns with testing large numbers of games with individuals with impairments, and this toolkit is not intended to replace effective, directed user testing. Instead it offers a low-cost, reasonably quick way to highlight

\footnotetext{
${ }^{1}$ https://tinyurl.com/meeplelikeus.
} 
important issues that must be considered before a gaming group considers a title for inclusion at the table. The limitations of this approach are discussed in Heron et al. (2018).

\section{Visual Accessibility}

Of all the issues considered in terms of accessibility, colour blindness is the one that it easiest to deal with and most consistently ignored. However, in this category of the toolkit we assess a wide variety of elements that will impact on visual impairment. Our recommendations primarily work on the assumption that we are not dealing with total blindness but instead on one of the more common visual impairments that permit some degree of visual differentiation with appropriate support.

Table 1 shows the elements of the toolkit that relate to those with visual impairments.

\section{Cognitive Accessibility}

For cognitive impairments, it is usually difficult to generalise feedback because of how tightly dependent an analysis is upon the specifics of rules, player agency, and the way the game flows. As such, the lenses in this section of the toolkit are intended to direct attention towards contemplation of the game as a whole, and how its inherent uniqueness reflects traits most likely to be cognitively demanding, as shown in Table 2.

In this category, we make use of a melding of fluid and crystalised intelligence (Cattell 1963) for discussion, although in the teardowns we rate them separately. This is in recognition of the tight coupling that exists between these concepts, and how difficult it is to unpick the impact of one on the other. The grading given for separate components represents a rough judgement of the relative burden the game may place on each.

\section{Emotional Accessibility}

While games are primarily intended as recreational activities, there can be a considerable disparity in terms of what different players may consider 'fun'. Some enjoy the thrill of winning, some enjoy the mastery of a puzzle, and others enjoy deceiving and fooling their friends. Some even enjoy the comedy that may come from a hilariously bad loss. There are numerous emotional triggers that may be associated with board games, and those elements considered as potentially triggering are outlined in Table 3: 
Table 1 Visual Accessibility

\begin{tabular}{|c|c|}
\hline $\begin{array}{c}\text { Choice of } \\
\text { colours }\end{array}$ & $\begin{array}{l}\text { Colour choice is an important consideration for those impacted by colour-blindness, } \\
\text { and it is often the case that games will make use of colour palettes that are } \\
\text { inaccessible to those wth Protanopia, Deteuronopia or Tritanopia. These problems } \\
\text { may exist in the form of player token choices, the board state itself, coloured cards, or } \\
\text { in tracking score. There will often be 'accessible combinations' of tokens that can be } \\
\text { used when dealing with lower player counts, but overlaps in the palette when dealing } \\
\text { with larger numbers }\end{array}$ \\
\hline Contrast & $\begin{array}{l}\text { Contrast represents a significant issue for those with visual impairments, and ensuring } \\
\text { effective contrast should be observed at all times in the game. We would traditionally } \\
\text { expect a minimum colour ratio of } 4.5: 1 \text { for normal sized text and } 3: 1 \text { for text of font } \\
\text { size } 14 \text { or higher, as per the Web Content Accessibility Guidelines (World Wide Web } \\
\text { Consortium 2008) }\end{array}$ \\
\hline
\end{tabular}

Font choice Fonts should ideally be chosen for readability, with a minimum of ornamentation. For maximum readability fonts should be bold, and make use of italics or ALLCAPS
Tactility of Often, games provide tokens which are impossible to differentiate by touch-they may tokens have many tokens of the same form factor permitting only visual differentiation. We recommend that games adopt the same principles for tactile differentiation that is in common usage for coinage (Schillmeier 2007)

Binocularity Some games require the ability to differentiate based on distance and perspective. These games are often dexterity based titles, and as such it's not easy to offer generalised compensatory guidance

Paper money Paper money is almost uniformly inaccessible in board games, lacking as it does even the slim tactile differentiation that real paper currency offers. The usual compensatory regimes for those with visual impairments (such as the folding method (Schillmeier 2007) are rarely appropriate when dealing with games. The rapidity through which currency may be circulated and the sums of money involved do not scale well to these techniques. Avoiding paper money is best, and if a game makes use of it we tend to recommend players adopt some other form of tracking wealth such as poker chips, matchsticks, or even actual money

Non standard Visually impaired gamers will often have invested in replacement dice. These may be dice over-sized, or even have their faces represented in braille. Dice of these kinds exist in all flavours, including the game specific varieties such as four, eight, ten, twelve and twenty sided dice. However, when a game includes non-standard dice faces as part of its provision, it's necessary for gamers to develop their own lookup tables before play begins. Providing these lookup tables for players can ease this transition although there will usually be a significant impact on flow of play until the tables are memorised

Indicative teardowns that show the impact of visual impairments on games may be found on Meeple Like Us-specifically the games Dixit; Lords of Waterdeep; Tales of the Arabian Nights and Galaxy Trucker. Examples of good design for visual accessibility may be seen in the teardowns for Patchwork, Paperback, Lanterns, and Splendor

\section{Physical Accessibility}

Almost every tabletop game requires a degree of physical interaction, whether it is moving pieces or shuffling cards. In this category, we assess the degree to which individuals with physical impairment might actually be able to enact such moves on their own behalf. We take into account how much movement is required, how often, over how large a space, and with what degree of precision. However, we also 
Table 2 Cognitive Accessibility

\begin{tabular}{|c|c|}
\hline Reading level required & $\begin{array}{l}\text { We work on the assumption that there is no need for a player to read the manual for } \\
\text { the game-that they will have the rules explained to them by a knowledgeable } \\
\text { party. However, the amount of text required on cards in play, and the complexity of } \\
\text { the instructions on them must be taken into account }\end{array}$ \\
\hline
\end{tabular}

Game state complexity Meaningful play in board games depends on evaluating a game state and making decisions based on the existing state to change it to a more desirable state. The complexity of a game will determine the cognitive costs needed to play. It's not intended that this be resolved down to a numerical value, such as 'equivalent mental age', since this is neither useful nor respectful

Memory requirements Most modern games do a good job of limiting the need for significant burden on memory. They allow for discarded cards to be examined at any time, provide cheat sheets that show key rule outcomes, remind players of their available actions, and so on. However, some games require players to hold certain elements of the game state in their head in order to make informed decisions for the future. We must be mindful of how much is required of a player if a game is to be cognitively accessible

Game flow

The consistency of game flow is an important element of learnability. Some games have a common structure that can be relied upon. Others have a turn order, and even turn composition, that may vary widely depending on what has happened within the game. Players may miss turns, or go several times in a row, or play may be executed in reverse order, or any combination of these. The more malleable the game flow is, the greater the cognitive burden that goes into understanding what is going on

Number of token combinations

Synergy of rules

Scoring

General knowledge/ trivia

Multitasking
Many games make use of cardboard, paper or plastic tokens to represent certain elements of game state. They may represent a player presence on a board, the availability of a particular resource, the scoring implications of a certain action, and so on. The more of these of which a game makes use, the greater the memory burden

It is a common trait of many games to offer rules synergy, which refers to the degree to which particular rules within the game amplify, or nullify, other rules. These may include simple synergies such as 'If you have played card $\mathrm{X}$, then card $\mathrm{Y}$ is at double strength', or more complex chains such as 'With card A in play, it means that card B is at half strength, which means that card C prevents card B from destroying card D, which means...'

The more synergistic combinations of rules that exist, the more cognitively expensive the game is to understand and play

Some games make use of complex scoring regimes that are designed to obscure the status of winning players until the end. This is designed to make sure that no-one feels disheartened by game progress. The more obfuscated and deferred these scoring systems may be, the more cognitively expensive it is for players to assess what their sensible actions are for current play

Some games are dependent on a degree of historical or general knowledge to support play. They may implicitly make use of cultural references, rely on others to understand geopolitical context, or need players to be able to answer questions of trivia. Such games place significant burdens on both recall and recognition, which can impose significant burdens on both fluid and crystallised intelligence

Many games require players to keep track of many competing goals and systems, and how those systems may act in concert to bring about a particular goal. The extent to which this is required plays a significant role in influencing cognitive accessibility

Relevant teardowns considering this category of cognitive accessibility may be found on Meeple Like Us. Specifically, Pandemic; Concordia; Dominion, Once Upon a Time, the X-Wing Miniatures Game; Star Fluxx; and Suburbia 
Table 3 Emotional Accessibility

Challenge

Despair

Arbitrary fates

Bluffing/lying

Need for closure/ symmetry

'Take That' mechanics

Upsetting themes

Score disparity

Player elimination

'Ganging up'
Much of the enjoyment of a game for many players comes from the mastery of a challenge, perhaps after multiple failures. However, challenge goes hand in hand with frustration (Juul 2009) and if the challenge of a game is too high it may end up being an issue

Several modern games adhere to the form sometimes known as 'despair generators'they are designed to offer extremely high challenge with the expectation of failure. The fun comes from simply enjoying the way that failure unfolds. However, it can often be hard to convince anyone that an embarrassing loss was actually fun, and this has to be taken into account

Games work best for many players when they represent largely controllable systemsthe extent to which a player is responsible for their own fate can be a powerful trigger for emotional upset. High degrees of randomness, or being set up with an unwinnable game state can impact on this. However, this is highly dependent on individual response. Some may find that it helps being able to dismiss a loss as being the luck of the draw. Others may interpret a loss that they themselves engineered as a reflection their own capabilities, and spiral accordingly

Many games involve a degree of bluff or outright lying- often through misleading other players as to their game state, or actively hinting at intentions for play which are false. For those with certain emotional conditions, it can be difficult to read the players around them and difficult to effectively manage this element of the gameplay

Some games will introduce a new game state on a turn by turn basis, or have a game structure that heavily implies an expectation of completion that cannot actually be achieved. Players may be able to 'finish' a task, but be penalised in gameplay terms for the attempt. This can exacerbate conditions such as OCD

A common element of a certain category of game is the 'take that' mechanic. This is a player action that can be performed after another player has taken their action, with the intention of countermanding it. The availability of such mechanisms is usually secret and highly conditional, and can significantly derail a player's strategy. The presence of such mechanics, and the gameplay weight associated, is an important element to consider in terms of the overall emotional impact of the game

There are many family games that exist, but just as many with 'mature' themes aimed at older gamers. Some games may make free reference to modern illegality or immorality, or reference particularly problematic elements of geopolitical context such as slavery. Some games may require players to act against type in playing, which can be an upsetting experience if there is no opportunity to avoid it. Such games may not be appropriate for families, or those with incompatible moral codes, regardless of the age rating on the box

The extent to which a player 'loses' is an important consideration in terms of how emotionally damaging a loss may be. If a player loses by a couple of points, it can be a case of simply congratulating someone on their win. If the win is by several hundred points, it's often hard for players to interpret that as anything other than a sign of their own stupidity. The extent to which a game enables large score disparities must be considered in this category

Some games allow for players to be knocked out of play, meaning they have to watch everyone else have fun until the game ends. This can understandably feel like exclusion, and exacerbate emotional upset

When a player develops an early lead in a multi-player, competitive game it makes sense from a game theory perspective for other players to focus negative attention upon the leader. The extent to which a game permits, or perhaps even encourages, ganging up on a single player is going to have a significant impact on the emotional suitability of the game

Relevant teardowns for this category include Star Fluxx; Galaxy Trucker; Merchants and Marauders; Tales of the Arabian Nights; Lords of Waterdeep; Pandemic; Patchwork; and Cards Against Humanity 
Table 4 Physical Accessibility

Size of cards

Token shape

Regularity of piece manipulation

Ease of communicating instructions

Physical acting

Paper money

Number of tokens

Size of game board elements
Some games make use of unconventional card forms, either extra-large or extra-small. Card manipulation of any kind may be difficult for those with physical impairments, but these non-standard cards can be especially difficult to work with

The degree to which tokens in the game permit easy manipulation is an important element of physical accessibility that must be taken into account. Tokens that are entirely smooth or rounded or are small and difficult to manipulate are going to cause problems for those with physical accessibility considerations. And often, with everyone else

The number of times a physical interaction is required is going to be a compounding factor on interaction difficulties. That which may be possible on an incidental basis may offer accessibility barriers if it must be repeated several times per minute. This includes moving tokens, shuffling cards, and interacting with shared communal game elements

Where there is a barrier to direct interaction with a game state, a substitute can be for impaired players to issue instructions verbally. How effective this is though depends on the ease of referring to particular areas of the game state and the extent to which the judgement of another player will come into executing the instruction

Some games, the most obvious of which is Charades, require a degree of physical acting or physicality of game state. Depending on the nature of this physical acting, it may render the game inaccessible to certain groups of players. Other games include the assumption of ability in the instructions, such as 'indicate that you are a spy by holding up your thumb'

Just as paper money is a problem for those with visual impairments, it is an interaction barrier in terms of physical accessibility. Paper money is more difficult to manipulate than other forms of currency representation, and this can be a problem for everyone, not just those with physical impairments

The busyness of a game board is going to be a significant determining factor in how easily physical manipulation can be handled. If working with lots of tokens in a small region of the board, even relatively minor physical impairments may get in the way of effective play through knocking other pieces out of their original configuration

The size of the 'target zone' of board manipulation is a powerful indicator of physical accessibility. If it's small, then the precision of physical dexterity required to place tokens where they should go is going to be significantly increased. If it is large, or does not require fine positioning within constraints, the requirements are considerably reduced

Relevant teardowns include Galaxy Trucker; Patchwork; the X-Wing Miniatures Game; Survive: Escape from Atlantis; and Ticket to Ride: Europe

acknowledge that in cases where that may not be possible there is an alternative option of verbalising instructions for another player to perform. For this we need to take into account the degree to which the game enables another player to consciously or subconsciously 'cheat' when enacting the wishes of the other player. Table 4 outlines the facets of these we consider as part of this toolkit. 
Table 5 Communicative accessibility

\begin{tabular}{|c|c|}
\hline Reading level & $\begin{array}{l}\text { Some games make heavy use of complex text to handle contextual } \\
\text { instructions, whereas others can be played without reference to any written } \\
\text { content. The extent to which the game can be understood in its deployment } \\
\text { language is an important communication consideration }\end{array}$ \\
\hline Audibility & $\begin{array}{l}\text { Few games have explicit audio components, but some of them do exist- } \\
\text { these sounds normally originate from an external source such as a CD or } \\
\text { digital app. The nature of these audio signals, and the extent to which the } \\
\text { information they convey is indicated elsewhere, determines the suitability } \\
\text { of the game for those with hearing impairments. This element of the lens } \\
\text { also tends to be an issue in games where large amounts of strategy or } \\
\text { narrative must be communicated between members of a group }\end{array}$ \\
\hline Lying/bluffing & $\begin{array}{l}\text { Much of the lying and bluffing in games is based on confidence, verbal } \\
\text { fluency, and body language. For those with impairments in any of those } \\
\text { categories, the need to bluff or ascertain bluffing in others may be very } \\
\text { limited. Similarly, the need to bluff may put considerable pressure or stress } \\
\text { on those that do not feel comfortable with taking on the role }\end{array}$ \\
\hline $\begin{array}{l}\text { Communication of } \\
\text { strategy }\end{array}$ & $\begin{array}{l}\text { Numerous modern games adopt a co-operative style in which all players work } \\
\text { together to accomplish a common goal. For these games, the difficulty } \\
\text { tends to be very high to compensate for the lack of direct player } \\
\text { competition. As such there may be a need to communicate complex, precise } \\
\text { strategy in a context for which there may not be a lot of real-world } \\
\text { analogues. This may also require a degree of advocacy, where the } \\
\text { originator of a plan must defend its suitability in the face of queries, } \\
\text { critiques, or alternative proposals }\end{array}$ \\
\hline $\begin{array}{l}\text { Need for audible } \\
\text { communications }\end{array}$ & $\begin{array}{l}\text { Some games require players to express non-trivial sounds in order to } \\
\text { communicate game state or game intention. Where these could not be easily } \\
\text { translated into written text, a visual communication language, or relayed by } \\
\text { a supporting player, these need to be taken into account. For example, if } \\
\text { communication must be kept secret it might not be appropriate to consider } \\
\text { sign-language as an appropriate way of dealing with the requirement }\end{array}$ \\
\hline
\end{tabular}

Relevant teardowns include Once Upon a Time; Tales of the Arabian Nights; Pandemic and Sentinels of the Multiverse

\section{Communication}

For this, we consider communication beyond that implied by any social interaction - that which is required by the game to participate in the experience. Some games require players talk over strategy, or communicate intention, or ask questions, or read complex instructions. These all represent potential communication barriers to play, as outlined in Table 5 .

We assume in this category though that communication between players is largely a solved problem - that everyone involved has some combination of communication regimes that permits every day discussion to occur. These framework elements then relate to those complicating issues that may frustrate these regimes with regards to the specific playability of a game. 
Table 6 Socioeconomic Accessibility

\begin{tabular}{|c|c|}
\hline Inclusive artwork & $\begin{array}{l}\text { We take into account how inclusive the artwork for a game is, paying attention } \\
\text { to gender balance and where appropriate the balance of appropriate ethnicities. } \\
\text { Some games have a theme that would naturally preclude an inclusive roster, } \\
\text { but for many we assess whether any given person could look at the game and } \\
\text { think 'I can see people like me represented on the box, so this is a game for } \\
\text { people like me' }\end{array}$ \\
\hline $\begin{array}{l}\text { Sexism in art and } \\
\text { instruction }\end{array}$ & $\begin{array}{l}\text { Games have long been considered a hobby 'for the boys' and as such certain } \\
\text { games tend to play into the common stereotype of pitching themselves at } \\
\text { presumed straight men. This comes through in art choice, manual wording, } \\
\text { colour assignments, and occasionally in an assumption of masculinity in the } \\
\text { game manuals. We assess games for the extent to which they make use of } \\
\text { these gendered, cisnormative and heteronormative assumptions }\end{array}$ \\
\hline
\end{tabular}

Theme

The theme of certain games can be extremely challenging, and may include considerable portions which may be considered upsetting or triggering. Where games are likely to include these elements, we outline them and the extent to which they are core to the game experience. In this, we don't judge games for including this kind of content. In fact, we enjoy many of these games ourselves. We seek only to ensure that it is appropriately documented so that others can consider whether the game is suitable for its intended deployment

Player counts

One of the more meaningful things that must be taken into account here is 'cost per player', which will be important for those on a budget looking to maximise the benefit they get out of a game. Low player counts may not allow for all members of a group to play, whereas high player counts may make it difficult to bring to the table with enough people to make it worthwhile. The extent to which games scale up and down in terms of enjoyment to player count is also a consideration here

Cost

Board games are not particularly cheap, and when budget is a consideration it's important that people feel they are getting something worth the money. Board gaming is widely considered to be a 'luxury hobby', and as such the cost of the game rarely has any direct relationship to the quality of the gameplay. Some games too have an expectation of further investment- that you are in essence putting a down-payment on a larger investment in the hobby

Relevant teardowns include Lords of Waterdeep; The X-Wing Miniatures Game; Tales of the Arabian Nights; and Sentinels of the Multiverse

\section{Socioeconomic Accessibility}

The definition of accessibility shouldn't be just in terms of disabilities-it also needs to encompass a kind of 'sociological' accessibility. It's not just about 'this is a game that I can play' but 'this is a game that I think is relevant to me'. There are games that are inaccessible not because of their physical design, but because they have elements of representation, of content, or of tone that are off-putting.

We incorporate numerous socioeconomic elements into this framework as outlined in Table 6 . 
Table 7 Intersectional Accessibility

Physical/cognitive

Size of Cards/hands It might be possible for a player to hold a hand of cards easily, or possible to easily understand all the cards in their hand. When neither of these are possible it can create a difficult situation for working out what options a player may have available to them

Board complexity Many complex boards can be understood easily by those with only physical impairments, and those with cognitive impairments may be able to move around the board to inspect particular elements in isolation to temporarily limit complexity. This may not be possible when these impairments combine

Dice

Hidden hands

Agency

\section{Emotional/cognitive}

Downtime

Competition

Physical/visual

Token size

Placement of tokens

Board size

Cognitive/visual

Aesthetics

Symbolism

Physical/communicative

Communicated instructions
Randomness creates a cognitive burden as a result of expanding the state of possible outcomes. Dice also cause a physical accessibility issue when it comes to rolling them. When these compound, it can lead to situations where there is a disconnect between the numbers that came up and why they led to the outcome

The ability to manage hidden hands of card information can be frustrated by this intersectional category, since getting assistance from another player may reveal secret game state information in the process

We believe that accessibility of games requires more than simple 'observer' status or having someone make moves on your behalf. The extent to which physical and cognitive impairments intersect for a given game will impact on this

Downtime between turns can make it difficult to keep attention focused, and it can also create situations of cognitive burden as players try to work out what's going to happen on their own turn. If it turns out that something horrible is coming their way, the delay as they watch it make its way around the table can also be emotionally upsetting

Competition is a healthy part of many games, but it depends on the ability of each player to be able to emotionally or cognitively deal with the consequences

Many games require not only manipulation of tokens, but also the ability to perceive them in context on the game board

The extent to which pieces can be placed in context will depend on the size of the 'target area'

The larger a board is, the more it becomes necessary to incorporate a degree of physical movement into examining it. When physical and visual impairments intersect, this may not be possible

The aesthetics of the game, and the jargon implied by those aesthetics, can make instructions and outcomes difficult to read. This can significantly increase the cognitive cost of comprehending the game rules

The use of symbols on cards can act as an effective, and even perhaps readable, shorthand for complex concepts. However, remembering and interpreting symbols can place a significant burden on cognitive processes

Many games are playable with physical impairments if you are prepared to let another player make moves on your behalf. That's not possible if you cannot communicate your intention to the other player 
Table 7 continued

All

Time constraints

Ability to drop in/out

Length of game sessions
Time constraints on player actions never take into account compensatory regimes that may be involved in making a title accessible. This can create frustration or stress if such limits are strictly observed. If they are more flexible,

experimenting with time limits may significantly impact on game balance

All impairments exist on a spectrum, and the position an individual may occupy on that spectrum may change day to day and hour to hour. The extent to which a game permits someone to drop out of play without impacting everyone else is an important aspect of accessibility

Some games may last many hours, which is a window of engagement that is large enough to impact upon any number and combination of impairments. The longer a game is, the more likely it is to exacerbate physical, emotional, or cognitive discomfort

\section{Intersectional Accessibility}

One of the more important areas that must be considered when performing an effective teardown of the accessibility of board games is the degree to which intersectionality must be considered. The ability to verbalise instructions may not be useful to someone with a communication impairment. The use of symbols may be okay for someone with a visual impairment, but a problem if that is compounded by a cognitive impairment. The size of a hand of cards might be fine for someone with a physical impairment, but when compounded by a visual impairment it may become difficult to fully ascertain the context.

As such, we include an intersectional discussion of each game for which we perform a teardown, assessing the way certain issues may compound problems. For this, we consider the elements outlined in Table 7.

\section{Conclusion}

This heuristic toolkit does not replace traditional user testing-you are almost always going to get better results through sitting down and playing through games with impaired players. However, there are very significant costs that are associated with such user testing, especially since it involves getting groups of players, each ideally with a different suite of impairments, in a room at a specific time for a specific period. It involves teaching the game, many instances of playing the game, and careful observation and evaluation of the results. It requires an understanding of the subtlety of interpreting user feedback. It'll often involve remuneration of participation, and the flexibility to deal with changes in arrangements, often at short notice.

The framework we have put forward in this paper requires a single informed analyst, familiar with the nature of accessibility and the game in question. Documentation of a teardown may be a time-consuming process, but the act of building up the data set is one heavily derived from contemplation and 
consideration of elements in isolation and then in conjunction. The work of the Meeple Like Us project has combined the accessibility teardowns of twenty games with fully documented write-ups of each. The time investment is not trivial, but much lower than direct user involvement. This toolkit then can ideally be used as a preliminary evaluative pass over games. This would ensure that the common elements of accessibility have been considered before it is passed on to actual users for more nuanced feedback.

Accessibility in board games remains an underexplored topic in academic and professional literature. This work represents a first attempt to codify some guiding principles under which it might be consistently assessed. It is important to understand this represents only an initial exploration of the problem space. Adoption of the framework by designers and publishers of board-games would offer an important step forward in addressing accessibility and inclusion. However, a more sustainable solution will require the development of generalizable compensatory regimes that make use of the full gamut of digital and analogue technology available. We expect the work outlined in this paper to inform future research in this area.

\section{Compliance with Ethical Standards}

Conflict of interest On behalf of all authors, the corresponding author states that there is no conflict of interest.

Open Access This article is distributed under the terms of the Creative Commons Attribution 4.0 International License (http://creativecommons.org/licenses/by/4.0/), which permits unrestricted use, distribution, and reproduction in any medium, provided you give appropriate credit to the original author(s) and the source, provide a link to the Creative Commons license, and indicate if changes were made.

\section{References}

Carroll, J. (2002). Play therapy: The children's views. Child and Family Social Work, 7(3), 177-187.

Cattell, R. B. (1963). Theory of fluid and crystallized intelligence: A critical experiment. Journal of Educational Psychology, 54(1), 1.

Di Loreto, I., Lange, B., Seilles, A., Andary, S., \& Dyce, W. (2013). Game design for all: The example of hammer and planks. In International conference on serious games development and applications (pp. 70-75). Berlin, Heidelberg: Springer.

Dunn, W., Brown, C., \& McGuigan, A. (1994). The ecology of human performance: A framework for considering the effect of context. American Journal of Occupational Therapy, 48(7), 595-607.

Fiske, J. (2010). Understanding popular culture. Abingdon: Routledge.

Harrison, T. M., \& Barthel, B. (2009). Wielding new media in Web 2.0: Exploring the history of engagement with the collaborative construction of media products. New Media \& Society, 11(1-2), $155-178$.

Heron, M. (2012). Inaccessible through oversight: The need for inclusive game design. Computer Games Journal, 1(1), 29-38.

Heron, M. J. (2015). A case study into the accessibility of text-parser based interaction. In Proceedings of the 7th ACM SIGCHI symposium on engineering interactive computing systems (pp. 74-83) ACM.

Heron, M. (2016). Cultural integration and the accessibility of gaming. Computer Games Journal, 5(3), 91-94. 
Heron, M., Belford, P., Reid, H., \& Crabb, M. (2018). Eighteen months of meeple like us-An exploration into the state of board game accessibility. Computer Games Journal: Special Issue on Game Accessibility. TBD

Heron, M., Hanson, V. L., \& Ricketts, I. W. (2013). Accessibility support for older adults with the ACCESS framework. International Journal of Human-Computer Interaction, 29(11), 702-716.

Hromek, R., \& Roffey, S. (2009). Promoting social and emotional learning with games: It's fun and we learn things. Simulation \& Gaming, 40, 626-644.

ICV2 (2015). Hobby games market climbs to $\$ 880$ million. Internal communication. http://icv2.com/ articles/markets/view/32102/hobby-games-market-climbs-880-million.

Juul, J. (2009). Fear of failing? the many meanings of difficulty in video games. The Video Game Theory Reader, 2, 237-252.

Law, M., Cooper, B., Strong, S., Stewart, D., Rigby, P., \& Letts, L. (1996). The person-environmentoccupation model: A transactive approach to occupational performance. Canadian Journal of Occupational Therapy, 63(1), 9-23.

Lee, S., Chung, J. E., \& Park, N. (2015). Linking cultural capital with subjective well-being and social support the role of communication networks. Social Science Computer Review, 34, 172-196.

Lenhart, A., Kahne, J., Middaugh, E., Macgill, A. R., Evans, C., \& Vitak, J. (2008). Teens, video games, and civics: Teens' gaming experiences are diverse and include significant social interaction and civic engagement. Pew internet \& American life project.

Pon, A. K. (2010). My Wonderful Life: A board game for patients with advanced cancer. Illness, Crisis \& Loss, 18(2), 147-161.

Roeder, O. (2015). Crowdfunding is driving a \$196 million board game renaissance. Five thirty eight. http:// fivethirtyeight.com/features/crowdfunding-is-driving-a-196-million-board-game-renaissance/?

Schillmeier, M. (2007). Dis/abling spaces of calculation: Blindness and money in everyday life. Environment and Planning D: Society and Space, 25(4), 594-609.

Westin, T., \& Dupire, J. (2016). Design of a curriculum framework for raising awareness of game accessibility. In International conference on computers helping people with special needs (pp. 501-508). New York: Springer.

Willekens, M., \& Lievens, J. (2014). Family (and) culture: The effect of cultural capital within the family on the cultural participation of adolescents. Poetics, 42, 98-113.

World Wide Web Consortium. (2008). Web content accessibility guidelines (WCAG) 2.0. 